\title{
Thermomechanical study of TiNi shape memory alloy during low-cycling test
}

Elzbieta Pieczyska ${ }^{1}$, Stefan Gadaj ${ }^{1}$ Wojciech Nowacki ${ }^{1}$ and Hisaaki Tobushi ${ }^{2}$

${ }^{1}$ Institute of Fundamental Technological Research, Polish Academy of Sciences, Swietokrzyska 21, 00-049 Warsaw, Poland; fax 4822826 9815, tel 48228261281

${ }^{2}$ Department of Mechanical Engineering, AICHI Institute of Technology, Toyota 470-0392, Japan; fax 81056548 8555, tel 81056548 8121, epiecz@aitech.ac.jp

Keywords: shape memory alloy, superelastic deformation, temperature change

\section{(Oral presentation)}

In applications to sensors, actuators, robots or solid state engines, a shape memory element (SMA) is used as a working element that performs cyclic motions. In order to evaluate the reliability of the SMA elements, cycling deformation properties of the material are of key value. In this paper low-cycling properties of TiNi SMA were studied in terms of mechanical and thermal effects. To this end, ten subsequent cycles of loading - unloading with the constant strain rate of $10^{-2} \mathrm{~s}^{-1}$ were repeated in room conditions (above the SMA $A_{f}$ temperature) for the maximum strain value $9 \%$.

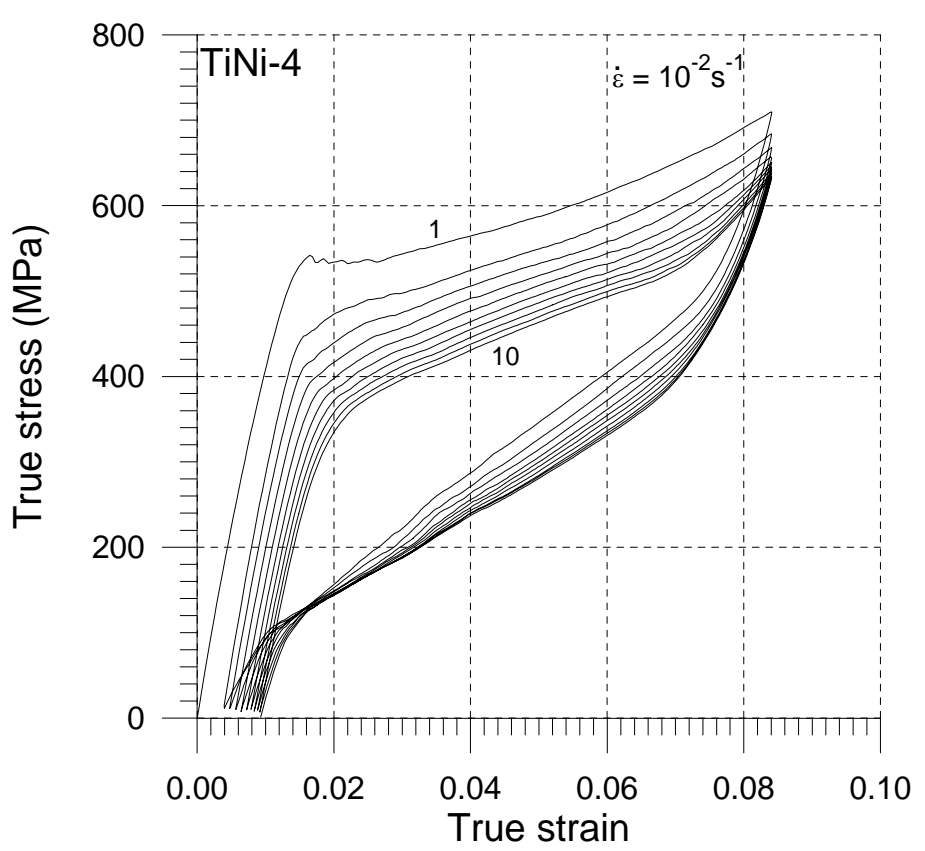

Figure 1. Stress-strain curves of TiNi SMA subjected to low-cycling test

The stress-strain curves obtained for the superelastic loading - unloading TiNi SMAs are presented in Fig. 1. One can notice that the run of the first cycle is similar to the stress-strain curve presented in [1] for the same strain rate. The yielding due to the martensite transformation occurs at a stress of $470 \mathrm{MPa}$ for strain above $1.5 \%$. For the subsequent cycles, the martensite stress decreases with the number of the cycle, 
however this amount of the decrease also decreases. The residual strain increases with the number of cycles, since both the residual martensite and microstructure defects are higher for the increasing number of cycles. The phenomena are related to temperature.

The temperature vs. true stress curves of the SMA subjected to this cycling test are presented in Fig. 2. For each cycle, an increase in temperature during the loading and the martensite transformation was observed followed by a significant temperature decrease accompanying the unloading process and the reverse transformation.

The temperature increment registered during the martensitic transformation for the first cycle was equal to $28 \mathrm{~K}$, while for the subsequent cycles of loading the temperature increments were significantly lower. The temperature decreases during the reverse transformation. After the reverse transformation was completed, the temperature drops below the initial temperature. For the subsequent cycles, the drops in temperature are even higher, however the saturation effects are also observed.

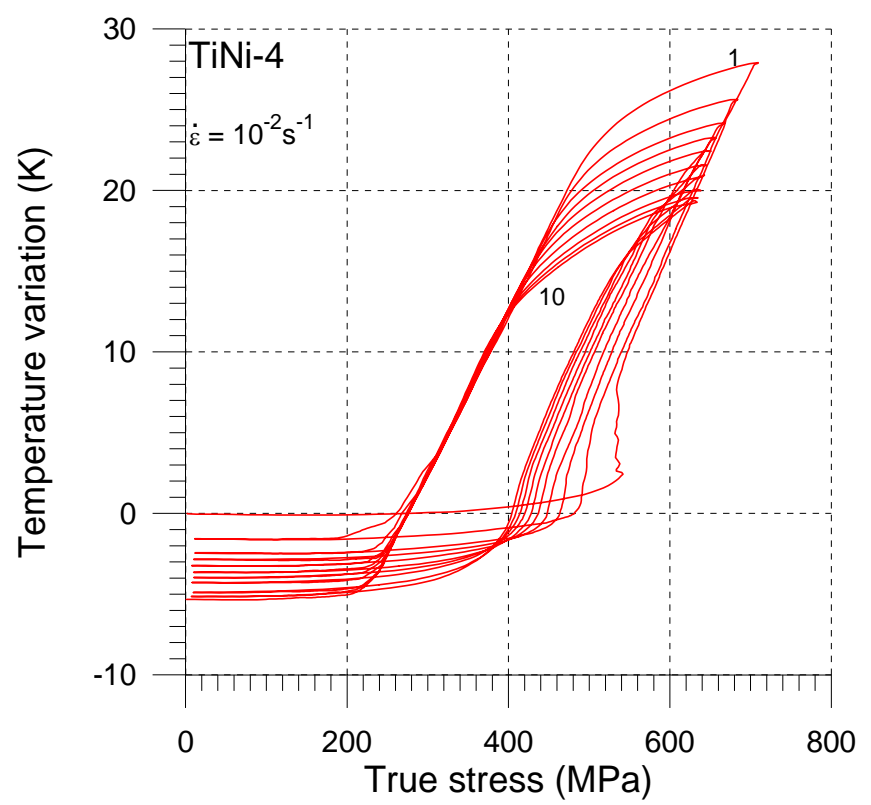

Figure 2. Stress and temperature curves of TiNi SMA subjected to subsequent cycling test with strain rate $10^{-2} \mathrm{~s}^{-1}$ at room temperature

Summarizing, the run of the mechanical and the temperature curves obtained during the TiNi SMA low-cling tests indicates that the temperature increments are mainly caused by the martensitic and the reverse transformations and in order to avoid too high the stress change and related to this too strong the temperature impact, the mechanical training before the material application is necessary.

References: 1) E. Pieczyska, S. Gadaj, W. Nowacki, Tobushi H., QIRT J. 1.1 (2004)

Acknowledgments: This research has been carried out under Grant No. 4 T08A 060 24, the JSPS Grants No: 13650104, Post-doc ID P04774, and Joint Research 6612. 